\title{
Morphological and Molecular Characterization of Marssonina coronaria (Ellis \& J.J. Davis) J.J. Davis Causing Premature Leaf Fall
}

\author{
S. Phurailatpam*, J.N. Sharma, A. Brakta, Sushma Sharma and Shweta Sharma \\ Department of Plant Pathology, College of Horticulture, Dr. Y.S. Parmar University of \\ Horticulture and Forestry, Solan173 230, Himachal Pradesh, India \\ *Corresponding author
}

\section{A B S T R A C T}

\begin{tabular}{|l|}
\hline K e y w o r d s \\
$\begin{array}{l}\text { Marssonina } \\
\text { coronaria, } \\
\text { Morphological, } \\
\text { Pathogenicity, ITS } \\
\text { regions }\end{array}$ \\
\hline Article Info \\
\hline $\begin{array}{l}\text { Accepted: } \\
\text { 20 May } 2019 \\
\text { Available Online: } \\
\text { 10 June } 2019\end{array}$ \\
\hline \hline
\end{tabular}

Marssonina leaf blotch caused by the fungus Marssonina coronaria (Ellis \& J.J. Davis) J.J. Davis causing premature leaf fall is one of the major economic constraint which hampers apple production in India. Seven isolates of Marssonina coronaria were isolated and evaluated for their morphological and molecular characterization using ITS primers. All the seven isolates were identified based on the morphological characteristics, and produced brown to black colonies without aerial mycelium, formed a wrinkled surface, on PDA after incubation for 30 days. Conidia are ampule shaped, one sepate, constricted at the septum, hyaline, and guttulate. However, not much difference was obtained in colony diameter and size of conidia. Seven isolates of the rDNA of ITS region was amplified by ITS4/ITS6 primers and ranged from 565 615 bp in the isolates. On BLAST, isolates showed high homologies (96 100\%) with Marssonina coronaria. Phylogentic tree depicted that the isolates were closely clustered with Chinese isolates with accession numbers FJ606800, EU329733, EU329735, FJ618562, FJ618561 and FJ618560. The present study demonstrated the genetic relationships among the isolates of Marssonina coronaria and indicated that ITS rDNA sequence data were potentially useful in taxonomic species determination.

\section{Introduction}

Marssonina leaf blotch caused by the fungus Marssonina coronaria (Ellis \& J.J. Davis) J.J. Davis causing premature leaf fall is relatively new threat to successful cultivation of apple. In India, this disease was first recorded in the year 1992 in Himachal Pradesh (Sharma et al., 2004). After it appearance, Marssonina Blotch appeared in epiphytotic form in Himachal Pradesh in 1996 and threatened the apple cultivation by causing premature leaf fall (Sharma and Kaul, 2000; Sharma, 2001) and it was so severe in some orchards that no foliage was left on the trees by the harvest time (Sharma and Gautam, 1997). It affects fruit size, colour, quality and fruit set adversely and reducing the tree vigour and fruit bearing capacity of the trees at the same time. Appearance of fruit spots on the produce makes it unsellable in the market leading to direct economic loss to the growers. The disease can be kept at low levels 
by following protective fungicidal spray at short intervals during the growing season. This practice is in vogue in India also, particularly in Himachal Pradesh, where protective fungicidal spray programme is adopted every year to keep the disease under check (Sharma and Gautam, 1997; Sharma, 1999). But this approach may often lead to fungicide wastage particularly when weather conditions are not congenial for disease development. On the other hand, however, favourable weather conditions might result in high buildup of the disease pressure making it compulsory to carry on fungicidal spray at short interval for effective disease control which will otherwise result in increased cost of production apart from added environmental pollution. Presently, this disease has turned out to be the most important disease in apple growing areas of India due to absence of resistant cultivars. All the available commercial cultivars of apple are susceptible to this disease (Sharma et al., 2010). The occurrence of this disease throughout the country prompted the gathering of information on the phylogenetic distribution of Marssonina species. The present study was conducted to study the morphological variation among different isolates collected and to determine the phylogenetic relationship of Marssonina isolates collected from different apple growing region of Himachal Pradesh and Uttarkhand based on the sequences of internal transcribed spacer (ITS)region.

\section{Materials and Methods}

\section{Collection and Isolation of Marssonina spp. isolates}

The infected apple leaf samples with young sporulating lesions showing characteristic symptoms of Marssonina blotch were collected from Kullu, Bilaspur and Shimla districts of Himachal Pradesh and Uttarkashi and Garwal districts of Uttarakhand, located at different altitudes ranging between 900 $2500 \mathrm{~m}$ above mean sea level, and brought to the laboratory and kept in refrigerator for further use. The fungi were isolated separately from collected leaves. Infected leaves lesions of Marssonina blotch including acervuli were cut into small pieces $(3-5 \mathrm{~mm})$ and surface sterilsed in $1 \%(\mathrm{v} / \mathrm{v})$ sodium hypochloride for 2 mins followed by three rinses with sterilized distilled water. After blotting dry with sterilsed filtered paper these bits were transferred to sterilized potato dextrose agar medium in sterilized petriplates. Bits were placed in each petriplates and incubated for 30 days at $25^{\circ} \mathrm{C}$. The isolates were purified through single spore isolation technique by transferring germinating conidia to petriplates containing sterilized PDA medium. For maintaining purity and virulence of the isolated pathogens, sub-culturing was done after every 20 days. The axenic culture was maintained at $4 \pm 1^{\circ} \mathrm{C}$ in refrigerator on PDA slants. The isolates from Himachal Pradesh were coded as Shimla1, Shimla2, Kullu1, Kullu 2 and Bilaspur and isolates from Uttarkhand were coded as UK 1 and UK2

\section{Morphological studies}

The colony morphology and colour of each isolate on PDA medium were examined after 30days of incubation. For sporulation the culture were maintained in 12 hour light and dark alternatively, then conidia were harvested from each isolate and mounted in water. The measurements of the spores or conidia were recorded with a calibrated ocular micrometer under compound microscope.

\section{Pathogenicity test under polyhouse conditions}

Pure culture of M.coronaria isolates was first multiplied on PDA medium and then spore suspension of fungus prepared by flooding the 
culture plate with sterilized distilled water. The fungal growth on Petri plate was scrapped with the help of a sterilized razor blade, strained through a double layer of sterile cheese-cloth into a $150 \mathrm{ml}$ flask and spore concentration adjusted to $5 \times 10^{4}$ conidia per milliliter with the help of a haemocytometer. Pathogenicity test was conducted on one year old healthy but highly susceptible potted apple plant under polyhouse conditions.

The leaves of potted plants were surface sterilized with 70 per cent ethanol and washed thoroughly with sterilized distilled water. These were sprayed with conidial suspension with the help of hand atomizer. The potted apple plants after inoculation with conidial suspension of the pathogen were immediately covered with the polythene sheets for 48 and $72 \mathrm{hrs}$ for incubation and maintenance of temperature and relative humidity for the appearance of the disease symptoms.

The uninoculated seedling sprayed with distilled water served as control. Observations on the development of symptoms and incubation period were recorded. The experiment was conducted on July when environmental conditioned were the most favourable for pathogen infection and natural incidence was prevalent.

\section{DNA Isolation}

For DNA extraction, 30 days old pure culture of each isolate of Marssonina spp. was used. The fungus colonies were scrapped with the help of a sterilized needle and were ground to a fine powder with mortar and pestle using liquid nitrogen, and DNA was extracted, according to standard protocols SaghaiMaroof et al., (1984). The genomic DNA was checked by agarose gel electrophoresis and stored at $-20^{\circ} \mathrm{C}$ for further use.
PCR amplification, sequencing and phylogenetic analysis

To characterize the unknown Marssonina sp. a region of the nuclear rDNA gene containing the ITS regions was amplified from the genomic DNA by PCR using the primer combinations ITS 4(5'-TCC TCC GCT TTA TTG ATA TGC 3')/ ITS 6(3-'GAA GGT GAA GTC GTA ACA AGG 5').PCR amplification was performed in a total volume of $25 \mu \mathrm{L}$ containing $0.5 \mu \mathrm{l}$ of Taq DNA polymerase, $2.5 \mu$ l Reaction buffer (1X),3.0 $\mu \mathrm{l}$ $\mathrm{MgCl}_{2} \quad(2 \mathrm{mM}), \quad 2.0 \mu \mathrm{l}$ Deoxynucleotide triphosphate (dNTPs) mixture was carried out in MJ Mini BIO-RAD personal thermal cycler-100 (PTC-100) with a total of 40 cycles. Each cycle consisted of: PCR conditions $94^{\circ} \mathrm{C}$ for $3 \mathrm{~min}$ (initial denaturation), $94^{\circ} \mathrm{C}$ for $1 \mathrm{~min}$ (denaturation), $55^{\circ} \mathrm{C}$ for $1 \mathrm{mins}$ (annealing) and $72^{\circ} \mathrm{C}$ for 2 min (extension). The total number of cycles was 40 , with the final extension of $72^{\circ} \mathrm{C}$ for 10min. The amplified products $(25 \mu \mathrm{l})$ were separated on $1 \%$ agarose gels in $0.5 \times \mathrm{TAE}$ buffer ( $\mathrm{pH}$ 8.0) in a gel electrophoresis apparatus (Biorad, USA) and electrophoresed for $30 \mathrm{~min}$ under constant voltage $(50 \mathrm{~V})$. The ethidium bromide (final concentration of 0.3 $\mathrm{mg} / \mathrm{ml}$ ) was added to the molten agarose at the time of gel casting. The gel was visualized on a UV trans-illuminator, and photographs were taken using a Gel Documentation System (Biorad, USA). A 100 plus bp DNA ladder (MBI, Fermentas) was used as molecular weight size markers. The PCR product were then purified and sequenced were sent to Xcleris Genomics, India under refrigerated conditions using gel packs and thermocol box. DNA sequencing was performed at the scientific solutions (Xcleris Genomics, India). Sequenced data so obtained was analyzed with the help of tools like BLAST (Basic Local Alignment Search Tool) (www.ncbi.nlm.nih.gov) for homology search and ClustalW. Phylogenetic analyses based in 
ITS regions were constructed using neighbour joining tree programme (PHYLIP 3.68).

\section{Results and Discussion}

\section{Morphological characteristics of the isolates}

The morphological characters presented in Table 1 revealed that colonies of all isolates were dark brown to black without aerial mycelium with colony diameter ranging from 9-14 mm which was maximum in Shimlal isolate and minimum in Bilaspur isolate. Conidia were hyaline, ampule shaped, one sepate, constricted at the septum, guttulate and conidia size ranged from 3-8 $\times 12-28 \mu \mathrm{m}$. On the basis of morphological characters the pathogenic fungus associated with Marssonina blotch of apple was identified as Marssonina coronaria. All the morphological characters of isolated fungus closely resembled to those described by earlier workers (Harada et al., 1974; Tamietti and Matta, 2003; Sharma et al., 2004; Lee et al., 2011).

\section{Pathogenicity test}

All the isolates were pathogenic and produced tiny yellow spots on the upper surface of leaves after inoculation. A considerable pathogenic variability was observed in incubation period among the isolates. Incubation period of isolates varied from 1220 days with minimum in Shimla 1 isolate (15days) followed by Shimla 2 (17days) and maximum in Bilaspur isolate (20days). Typical Marssonina blotch symptoms as natural occurrence in field were observed after 30-35 days of inoculation in all the isolates. All the isolates after inoculation appears as grayish brown leaf spots on mature leaves and often looks tinged purple at the periphery. Small black acervuli are often visible on the spots. These lesions coalesce and surrounding areas turn chlorotic followed by defoliation tiny yellow spots later enlarged to form grayish brown circular lesions (5$10 \mathrm{~mm}$ diameter). Reisolation of the identical fungus from the inoculated leaves completed Koch postulates. The pathogen cities of isolates were not visually different in their symptoms developed on inoculated leaves until 5-6 week after inoculation. Successful fungal infection depended moist incubation under controlled condition.

This result suggests that this fungus needs an extended moist period to produce a successful infection. This partially explains why this disease usually starts after rain and occurs mostly in June and July. The findings are in agreement with Lee et al., (2011) who reported that tiny yellow spots appeared on upper surface of leaves after 21 days of inoculation and typical Marssonina blotch symptoms were observed after 40 45 days of inoculation.

\section{Molecular characterization}

The ITS regions are amplified by ITS4/ITS6 primers ranged from $565 \sim 615$ bp in the isolates (Fig. 1). On BLAST, isolates showed high homologies (96 100\%) with Marssonina coronaria. The sequences were submitted to NCBI and its GenBank Accession Number were KM873722, KM873723, KM873724, KM873725, KM873726, KM873727 and KM87372. The isolate Shimla1 showed 100\% homology with Marssonina coronaria with accession number KM873722, Shimla 2 and Kullu 1 showed 97\% homology with accession number KM873723 and KM873724, Kullu 2 showed 98\% homology with accession number KM873725, Bilaspur isolate showed $99 \%$ homology with accession number KM873726, Similarly isolates UK 1 and UK 2 showed 98\% and 96\% homology with Marssonina coronaria with accession number KM873727 and KM873728 (Table 2). 
Table.1 Morphological variability among Marssonina coronaria isolates

\begin{tabular}{|l|l|c|c|c|}
\hline Isolates & \multicolumn{1}{|c|}{ Colony colour } & $\begin{array}{c}\text { Colony } \\
\text { diameter } \\
(\mathbf{m m})\end{array}$ & $\begin{array}{c}\text { Conidia } \\
\text { colour }\end{array}$ & $\begin{array}{c}\text { Conidia size } \\
(\boldsymbol{\mu m})\end{array}$ \\
\hline $\begin{array}{l}\text { Shimla } \\
\mathbf{1}\end{array}$ & Dark brown to black without aerial mycelium & 14 & Hyaline & $4-6 \times 12-20$ \\
\hline $\begin{array}{l}\text { Shimla } \\
\mathbf{2}\end{array}$ & Dark brown to black without aerial mycelium & 13 & Hyaline & $4-7 \times 14-21$ \\
\hline Kullu 1 & Dark brown to black without aerial mycelium & 12 & Hyaline & $3-5 \times 12-19$ \\
\hline Kullu 2 & Dark brown to black without aerial mycelium & 12.5 & Hyaline & $5-7 \times 13-22$ \\
\hline $\begin{array}{l}\text { Bilaspu } \\
\text { r }\end{array}$ & Dark brown without aerial mycelium & 9 & Hyaline & $3-6 \times 12-20$ \\
\hline UK1 & Dark brown to black without aerial mycelium & 12 & Hyaline & $5-7.5 \times 12-28$ \\
\hline UK2 & Dark brown to black without aerial mycelium & 13 & Hyaline & $6-8 \times 13-22$ \\
\hline
\end{tabular}

Table.2 Identity of Marssonina spp. isolates inciting Marssonina leaf blotch of apple in India

\begin{tabular}{|c|l|c|c|c|c|c|c|}
\hline $\begin{array}{c}\text { Sr. } \\
\text { No. }\end{array}$ & Isolates & $\begin{array}{c}\text { Gene bank acc } \\
\text { no }\end{array}$ & $\begin{array}{c}\text { Max. } \\
\text { score }\end{array}$ & Total score & Query cover & E value & Ident \\
\hline $\mathbf{1}$ & Shimla 1 & KM873722 & 1133 & 1133 & $100 \%$ & 0.0 & $100 \%$ \\
\hline $\mathbf{2}$ & Shimla 2 & KM873723 & 1022 & 1022 & $100 \%$ & 0.0 & $97 \%$ \\
\hline $\mathbf{3}$ & Kullu 1 & KM873724 & 1035 & 1035 & $100 \%$ & 0.0 & $97 \%$ \\
\hline $\mathbf{4}$ & Kullu 2 & KM873725 & 989 & 989 & $91 \%$ & 0.0 & $98 \%$ \\
\hline $\mathbf{5}$ & Bilaspur & KM873726 & 994 & 994 & $91 \%$ & 0.0 & $99 \%$ \\
\hline $\mathbf{6}$ & UK1 & KM873727 & 966 & 966 & $91 \%$ & 0.0 & $98 \%$ \\
\hline $\mathbf{7}$ & UK2 & KM873728 & 917 & 917 & $91 \%$ & 0.0 & $96 \%$ \\
\hline
\end{tabular}

Fig.1 Amplified PCR product of genomic DNA

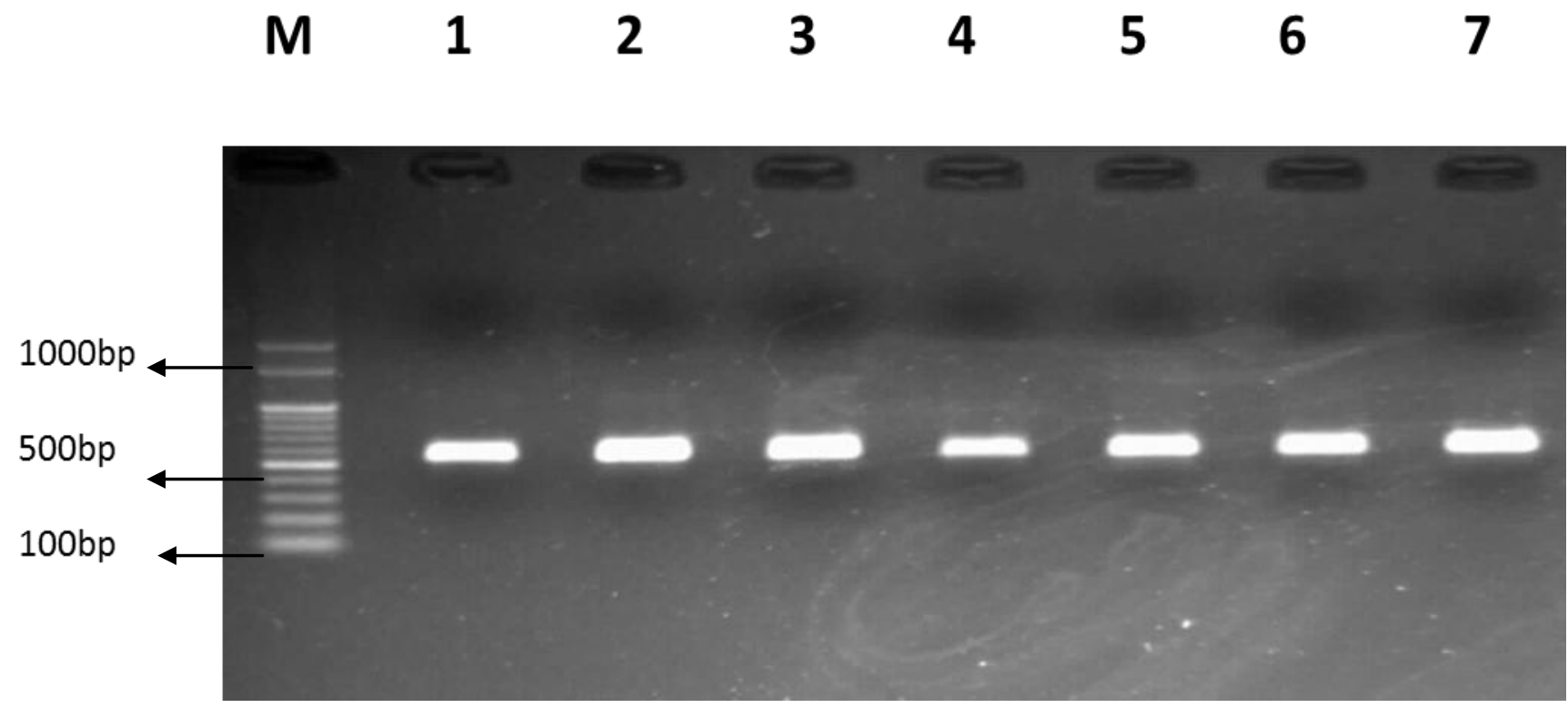


Fig.2 Phylogenetic tree based on the ITS sequences drawn using the neighbor-joining tree method showing relationship between the seven isolates of Marssonina coronaria

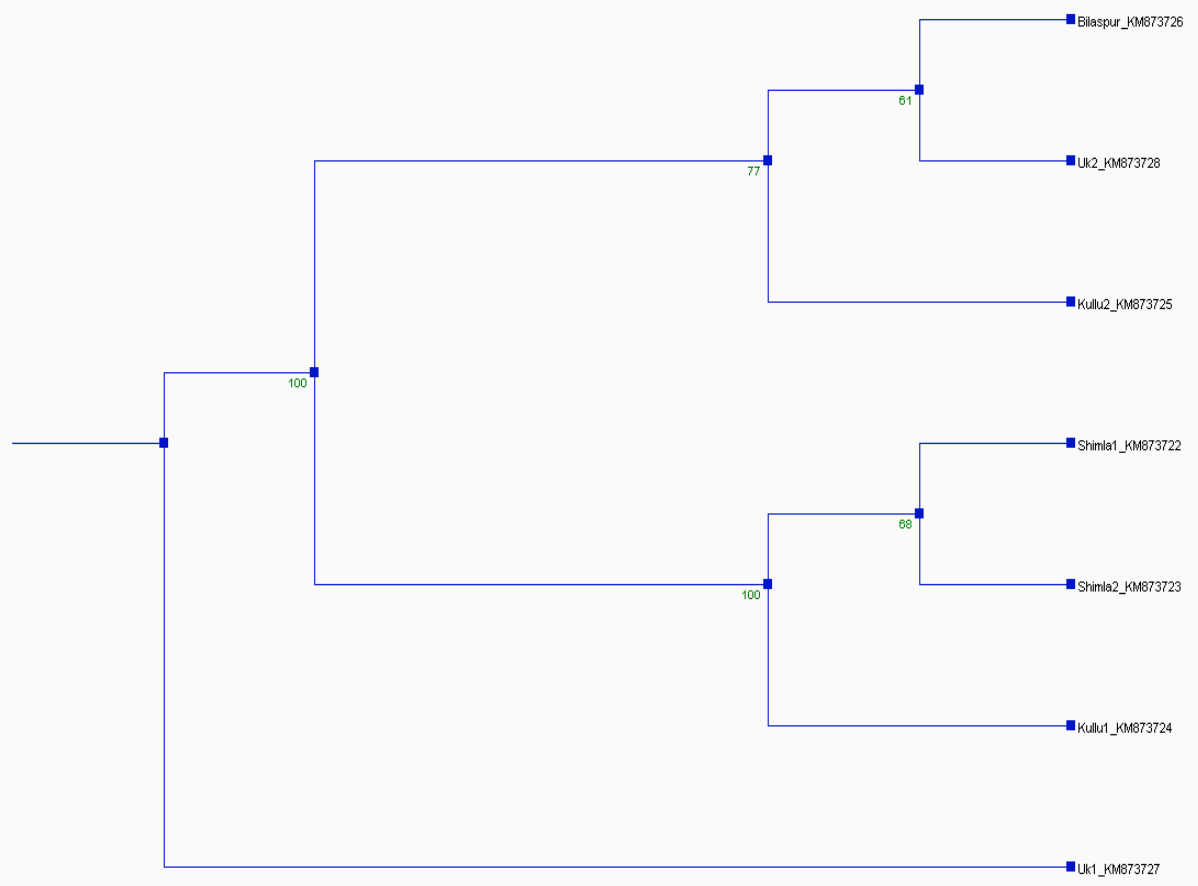

Fig.3 Neighbor-joining tree based on ITS gene sequences showing relationship between the 7 isolates of Marssonina coronaria and other sequences of Marssonina coronaria deposited in the NCBI Genbank

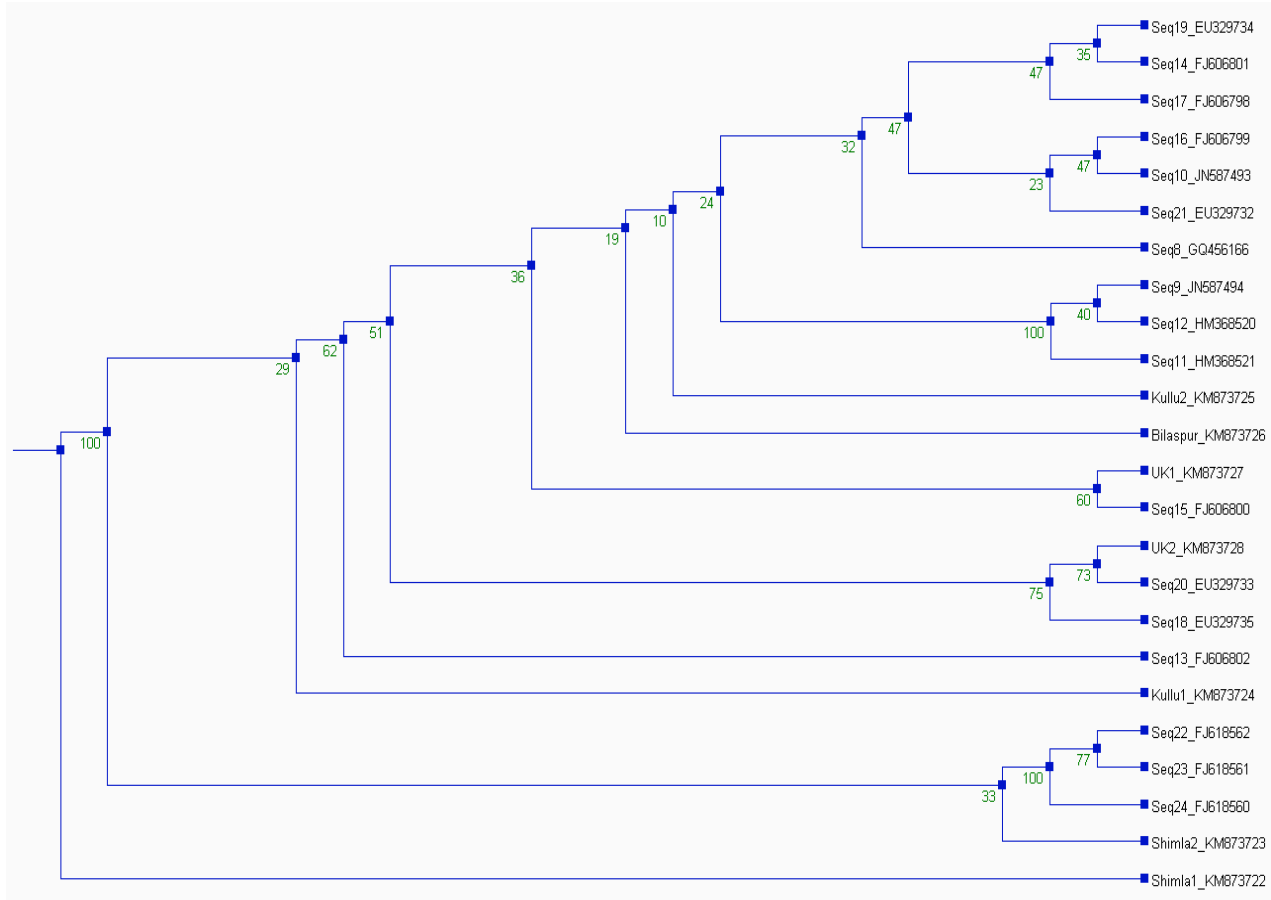


Therefore, little genetic diversity among $M$. coronaria isolates was indicated by ITS gene sequences

Phylogenetic tree was constructed using PHYLIP 3.68. All the sequences used for phylogenetic analysis were analyzed by TREEVIEW programme there in. The genetic relationship inferred from the nucleotide sequence polymorphism in the ITS region of the seven isolates are depicted in (Fig. 2). Two subclades were formed i.e. Group 1 and Group 2 with the major phylogenetic clades of Marssonina sp. First subclade contains the isolate UK1 which is different from rest of the isolates. No variability (maximum similarity) was found between the isolates (Bilaspur and UK2) and (Shimla 1 and Shimla 2).

Neighbor-joining method employed to construct the phylogenetic tree depicted the evolutionary pattern of the seven isolates of Marssonina coronaria and its relationship with ITS sequences of different Marssonina coronaria obtained from NCBI Genbank are depicted in Figure 3 and showed that UK 1, UK 2, Shimla 2 isolates was close clustered to Chinese isolates with accession no.FJ606800, EU329733, EU329735, FJ618562, FJ618561 and FJ618560.White et al., (1990) reported that ITS sequences are genetically constant or show little variation within species but vary between species in a genus. Thirteen isolates of Marssonina coronaria collected from Gyeongbuk Province from 2005 2007 displayed over $99.6 \%$ sequence similarity to each other in internal transcribed spacer regions and were phylogenetically closely related to Chinese isolates as reported by Lee et al., (2011).

From the present studies it is evident thatthe pathogen associated with Marssonina blotch belongs to Marssonina coronaria only, throughout Himachal Pradesh and Uttarakhand and showed genetic variation among the isolates collected from different locations. Starking Delicious, commercial cultivar of Himachal Pradesh is highly susceptible to Marssonina coronaria microflora available in the state, while Tydeman's Early Worcester showed moderately susceptible and Granny Smith moderately resistant reaction.

\section{References}

Burdon J.J. and Silk J. 1997.Sources and patterns of diversity in plant pathogenic fungi Phytopathology 31: 353-373.

Harada Y, Sawamura K and Konno K. 1974. Diplocarponmali, sp.nov, the perfect state of apple blotch fungus Marssonina coronaria. Annals of Phytopathological Society of Japan 40: 412-418.

Lee D H, Chang G B, Nang K K W, Kyung H C, Kyung M K, In KK, Cheol C, Yoon T M, Jae Y U and Hee Y J. 2011. Biological characterization of Marssonina coronaria associated with apple blotch disease. Microbiology 39: 200-205.

McDermott J M and McDonald B A.1993. Gene flow in plantpathosystem. Annual Review of Phytopathology 31: 353-373.

Saghai-Maroof M A, Soliman R A, Jorgensen R W and Allard A. 1984. Ribosomal DNA spacer-length polymorphism in barley: Mendelian inheritance, chromosomal location, and population dynamics. Biotechnology 14: 34-38.

Sharma I M and Bhardwaj S.S. 2003. Efficacy and economics of different fungicide spray schedule in controlling premature leaf fall of apple. Plant Disease Research 18: 21-24.

Sharma J N and Gautam D R. 1997. Studies on premature leaf fall of apple -a new problem. Journal of Plant Protection 25: 8-12.

Sharma J N and Kaul J L. 2000. Marssonina Blotch-a new disease hits apple crop in 
Himachal Pradesh. In: Proceedings of International Conference Integrated Plant Disease Management for Sustainable Agriculture. Indian Phytopathological Society, IARI, New Delhi, pp. 775-777.

Sharma J N, Sharma A and Sharma P. 2004. Outbreak of Marssonina blotch in warmer climates causing premature leaf fall problem of apple and its management. Acta Horticulturae 662: 405-409.

Sharma J N. 2001. Diagnosis and control of premature leaf fall problem in apple and its control. Journal of Mycology and Plant Pathology31: 305-31.

Sharma N, Thakur V S, Khurana S M P,
Mohan J and Sharma S. 2010.Variations in morphological parameters of apple cultivars in relation to premature leaf fall caused by Marssonina coronaria. Journal of Mycology and Plant Patholology 40(3): 454-459.

Tamietti G and Matta A. 2003.First report of leaf blotch caused by Marssonina coronariaon apple in Italy. Plant Disease 87 (8):1005.

White T J, Bruns T, Lee S. and Taylor J. 1990.Amplification and direct sequencing of fungal ribosomal RNA genes for phylogenetics. In: PCR Protocols: A guide to Methods and Applications. Academic Press, Orlando, Florida. pp. 315-322.

\section{How to cite this article:}

Phurailatpam, S., J.N. Sharma, A. Brakta, Sushma Sharma and Shweta Sharma. 2019. Morphological and Molecular Characterization of Marssonina coronaria (Ellis \& J.J. Davis) J.J. Davis Causing Premature Leaf Fall. Int.J.Curr.Microbiol.App.Sci. 8(06): 2762-2769. doi: https://doi.org/10.20546/ijcmas.2019.806.333 